\title{
PRODUÇÃO DE ZAMAC 2 POR MOAGEM DE ALTA ENERGIA E CONSOLIDAÇÃO POR CONFORMAÇÃO A QUENTE
}

\author{
Thais Alexandrino Proença' \\ Kamila Kazmierczak' \\ Júlio César Giubilei Milan ' \\ César Edil da Costa ${ }^{\prime}$
}

\section{Resumo}

O objetivo deste trabalho foi produzir a liga de Zamac 2 por metalurgia do pó através da moagem de alta energia e posterior conformação a quente. A caracterização dos pós foi realizada quanto à morfologia e à microestrutura, enquanto que os produtos consolidados foram caracterizados quanto a sua microestrutura, densificação final e dureza. A liga Zamac 2 foi preparada por meio da moagem dos pós elementares ( $\mathrm{Zn}, \mathrm{Al}, \mathrm{Cu}$ ) em moinho atritor operando a $400 \mathrm{rpm}$ com atmosfera de argônio e tempos de moagem variáveis. O produto obtido foi compactado uniaxialmente a frio $(400 \mathrm{MPa})$ e conformado a quente $\left(350^{\circ} \mathrm{C} / 500 \mathrm{MPa}\right)$. As micrografias mostraram que $10 \mathrm{~h}$ de moagem foram suficientes para atingir o estado de equilíbrio entre soldagem e fratura das partículas. O pó de Zamac 2 mostrou baixa compressibilidade devido ao aumento da resistência à deformação durante o processo de moagem de alta energia. Após a consolidação, a liga apresentou um aumento de $10 \%$ na sua densidade, boa coesão entre as partículas e distribuição homogênea das fases $\eta, \alpha$ e $\varepsilon$. A dureza da amostra conformada foi estatisticamente igual à da liga Zamac ZL4CZAP fundida.

Palavras-chave: Liga Zamac 2; Moagem de alta energia; Consolidação a quente.

\section{ZAMAK 2 ALLOY PRODUCED BY HIGH-ENERGY MECHANICAL MILLING AND CONSOLIDATED BY HOT PRESSING}

\begin{abstract}
The objective of this work was to produce, by high-energy milling, Zamak 2 alloy powder and consolidated it by hot pressing. The characterization of the powders was performed in terms of morphology and microstructure, while the consolidated products were characterized in terms of microstructure, final densification and hardness. Zamak 2 alloy powder was prepared by milling the elementary powders $(\mathrm{Zn}, \mathrm{Al}, \mathrm{Cu})$ in an attrition mil at $400 \mathrm{rpm}$, in argon atmosphere and varying times. The obtained powder was compacted $(400 \mathrm{MPa})$ and then hot pressed $\left(350^{\circ} \mathrm{C} / 500 \mathrm{MPa}\right)$. The micrographs showed that $10 \mathrm{~h}$ of milling were sufficient to reach the state of equilibrium between welding and fracture. Zamak 2 powder showed low compressibility due to the increased deformation resistance during the high-energy milling. After consolidation, the alloy presented a $10 \%$ increase in density, good cohesion between the particles and homogeneous distribution of $\eta, \alpha$ and $\varepsilon$ phases. The hardness of the consolidated sample was statistically equal to that of the Zamak ZL4CZAP casting alloy. Keywords: Zamak 2 alloy; High-energy milling; Hot pressing.
\end{abstract}

\section{INTRODUÇÃO}

As ligas à base de zinco e alumínio exibem propriedades físicas e mecânicas atraentes, como ponto de fusão relativamente baixo - cerca de $400^{\circ} \mathrm{C}$, excelente fundibilidade, boa resistência à corrosão e ao desgaste [I]. Por esses motivos, essas ligas podem ser usadas em várias aplicações de engenharia, como produção de engrenagens, polias e mancais de deslizamento [2].
Como resultado de diversas pesquisas nas últimas décadas, um grande número de ligas comerciais de zinco e alumínio foi desenvolvido, dentre as quais, destaca-se a família Zamac, que contém teor de alumínio inferior a 5, $1 \%$ em peso e apresenta uma excelente combinação de custo, ductilidade e resistência ao impacto [3].

'Programa de Pós-graduação em Ciência e Engenharia de Materiais - PPGCEM, Laboratório de Metalurgia do Pó, Universidade do Estado de Santa Catarina - UDESC, Joinville, SC, Brasil. E-mail: thaisaproenca@gmail.com

2176-1523 (C) 2019 Associação Brasileira de Metalurgia, Materiais e Mineração. Publicado pela ABM. Este é um artigo de acesso aberto distribuído sob os termos da licença Creative Commons CC BY-NC-ND (Attribution-NonCommercial-NoDerivs) - https:// creativecommons.org/licenses/by-nc-nd/4.0\%. 
Entre as ligas da família Zamac, a Zamac 2 é a que apresenta maiores dureza, resistência à tração e à fadiga [4]. Essa liga é também a que contém maior teor de cobre ( $3 \%$ em peso) - elemento que apresenta baixa solubilidade no zinco e, por isso, quando é adicionado em quantidades maiores que $1,25 \%$ em peso, precipita-se na forma do composto intermetálico $\mathrm{CuZn}_{4}$ (fase $\varepsilon$ ), ocasionando a expansão dimensional da liga [5].

Uma das formas de minimizar essa variação dimensional é utilizar tratamentos térmicos que acelerem a precipitação da fase $\varepsilon$ e promovam sua decomposição na fase T' $\left(\mathrm{Al}_{4} \mathrm{Cu}_{3} \mathrm{Zn}\right)$, que é estável [6]. Para essa finalidade, pode-se usar, por exemplo, o tratamento térmico T6 - composto por etapas de solubilização, têmpera e envelhecimento artificial. De maneira alternativa, Silva e colaboradores [7] obtiveram a fase T' ao produzirem a liga Zamac 2 por meio da moagem de alta energia dos pós elementares em moinho planetário por 30 horas. Segundo os autores, em elevados tempos de moagem, há - aumento da energia de deformação e da temperatura, os quais favorecem a difusão do alumínio no cobre e a formação da fase T'.

A moagem de alta energia é uma técnica pertencente à metalurgia do pó que apresenta diversas vantagens em relação aos métodos tradicionais, tais como o controle exato da composição química do produto final, a redução ou eliminação das operações de usinagem, a pureza dos produtos obtidos e a facilidade de automação do processo produtivo [8].

Diante do exposto, o objetivo desse trabalho foi produzir, por meio da moagem de alta energia em moinho atritor, o pó da liga Zamac 2 e caracterizá-lo quanto à sua morfologia e sua microestrutura. Adicionalmente, foram quantificadas a densificação final, a microestrutura e a dureza de amostras de pó conformadas a quente.

\section{MATERIAIS E MÉTODOS}

Pós elementares de zinco, alumínio e cobre, cujas proporções e características são dadas na Tabela I, foram submetidos à moagem de alta energia em moinho atritor por 2, 6 e 10 horas. Os parâmetros usados no ensaio de moagem são descritos na Tabela 2.

Utilizou-se atmosfera de argônio durante a moagem para prevenir a oxidação excessiva dos pós e adicionou-se 0 agente de controle de processo para minimizar a soldagem a frio dos pós uns com os outros e com a parede do tanque de moagem.

A morfologia e a microestrutura dos pós foram analisadas através da microscopia eletrônica de varredura com emissão de campo (FEG, JEOL-JSM 6 I07F, Tóquio, Japão). Para observar a microestrutura, os pós foram preparados metalograficamente e atacados com reagente de Palmerton ( $50 \mathrm{~g} \mathrm{CrO}_{3}, 4 \mathrm{~g} \mathrm{Na}_{2} \mathrm{SO}_{4}$ e $1000 \mathrm{~mL} \mathrm{H} \mathrm{H}_{2}$ ).
Com o objetivo de se obter doze amostras cilíndricas $(\phi=25 \mathrm{~mm}, \mathrm{~h}=6 \mathrm{~mm})$ para serem usadas na conformação a quente, compactou-se uniaxialmente a frio (400 MPa) cerca de $10 \mathrm{~g}$ de pó de Zamac 2 por amostra. Após essa compactação, as amostras obtidas foram, uma a uma, conformadas a quente.

O processo de conformação a quente consistiu no aquecimento de cada amostra por 30 minutos a $350^{\circ} \mathrm{C} \mathrm{em}$ uma matriz cilíndrica, a qual era mantida aquecida por um forno elétrico. Após 30 minutos, aplicou-se, com o auxílio de um punção, $500 \mathrm{MPa}$ de pressão, retirando-se em seguida a amostra conformada, a qual foi resfriada a temperatura ambiente. A Figura I mostra a disposição da amostra no sistema matriz-punção utilizado, bem como a montagem da matriz, do forno e do termopar sobre a prensa hidráulica.

As densidades das amostras compactadas e conformadas a quente foram calculadas através da Equação I, onde $\rho_{t}$ é a densidade teórica da Zamac 2, indicada pela ASTM B86-I 3 [4]. A massa $(m)$ das amostras foi medida em uma balança de precisão $( \pm 0,00 \mathrm{I} g)$, enquanto que a altura $(\mathrm{h})$ e o diâmetro (d) foram medidos com um paquímetro $( \pm 0,01 \mathrm{~mm})$.

$$
\text { Densidade }(\%)=\frac{4 \cdot m}{\Pi \cdot d^{2} \cdot h \cdot \rho_{t}} \cdot 100
$$

A microestrutura da amostra conformada a quente foi analisada por meio de microscopia eletrônica de varredura (MEV-FEG). As amostras foram preparadas pelo método convencional de microscopia - corte transversal, lixamento, polimento com solução de alumina e ataque químico com Nital 3\%.

A microdureza foi medida em um microdurômetro (Shimadzu HVM-2T) com carga de 0,5 kg por $10 \mathrm{~s}$. $O$ resultado obtido foi comparado com a microdureza da liga Zamac ZL4CZAP fundida (Zincoligas Ltda), cuja proporção dos principais elementos $(\mathrm{Zn}, \mathrm{Al}, \mathrm{Cu})$ é muito similar à da Zamac 2 (Tabela 3), justificando a sua utilização como parâmetro de comparação.

Tabela I. Proporções e características dos pós elementares

\begin{tabular}{ccccc}
\hline Material & $\begin{array}{c}\text { Quantidade } \\
\text { (\% p) }\end{array}$ & $\begin{array}{c}\text { Tamanho } \\
\text { de } \\
\text { partícula }\end{array}$ & Pureza & Fabricante \\
\hline $\mathrm{Zn}$ & 93 & $\leq 150 \mu \mathrm{m}$ & $99,83 \%$ & Synth \\
$\mathrm{Al}$ & 4 & $\leq 44 \mu \mathrm{m}$ & $99,7 \%$ & Alcoa \\
$\mathrm{Cu}$ & 3 & $\leq 53 \mu \mathrm{m}$ & $99,5 \%$ & Metalpó \\
\hline
\end{tabular}

Tabela 2. Parâmetros usados para a moagem de alta energia

\begin{tabular}{|c|c|}
\hline Condição & \\
\hline Equipamento & $\begin{array}{c}\text { Moinho atritor } \\
\text { (Union Process 0I-HD attritor) }\end{array}$ \\
\hline Bolas & AISI- $420, \phi 10 \mathrm{~mm}$ \\
\hline Velocidade de rotação & $400 \mathrm{rpm}$ \\
\hline Atmosfera & Argônio de alta pureza $(99,99 \%)$ \\
\hline Razão bolas-pó & $20: 1$ \\
\hline Agente de controle de processo & I,5\% p ácido esteárico \\
\hline
\end{tabular}





Figura I. (a) Vista frontal do sistema matriz - punção montado; (b) Montagem do forno, termopar e sistema matriz-punção sobre a prensa hidráulica.
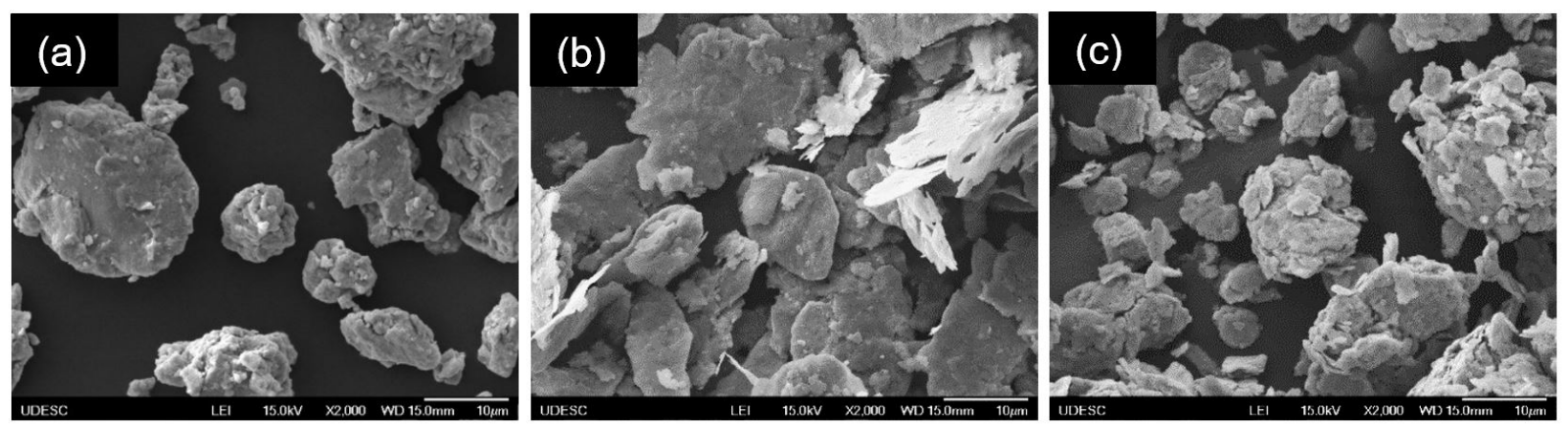

Figura 2. Evolução da morfologia do pó de Zamac 2 durante o processo de moagem: (a) 2 h, (b) 6 h, (c) 10 h.

Tabela 3. Comparação da composição química da liga Zamac ZL4CZAP com a da Zamac 2

\begin{tabular}{cccc}
\hline \multirow{2}{*}{ Elemento } & \multicolumn{2}{c}{ Zamac ZL4CZAP } & \multirow{2}{*}{ Zamac 2 } \\
\cline { 2 - 3 } & Mínimo & Máximo & \\
\hline $\mathrm{Al}$ & 3,500 & 4,200 & 4,000 \\
$\mathrm{Cu}$ & 2,000 & 2,700 & 3,000 \\
$\mathrm{Mg}$ & 0,450 & 0,650 & - \\
$\mathrm{Fe}$ & 0,000 & 0,035 & - \\
$\mathrm{Zn}$ & 92,404 & 94,050 & 93,000 \\
\hline
\end{tabular}

\section{RESULTADOS E DISCUSSÃO}

A variação da morfologia do pó de Zamac 2 durante 2,6 e $10 \mathrm{~h}$ de moagem é mostrada na Figura 2. Após $2 \mathrm{~h}$ de moagem, as partículas encontram-se achatadas devido às forças compressivas entre as partículas e as bolas de moagem (Figura 2a). Aumentando o tempo de moagem para $6 \mathrm{~h}$, prevalece a soldagem e quebra das partículas, porém ainda existe um grande número de partículas planificadas (Figura 2b). Com 10 horas de moagem, as partículas são equiaxiais, indicando que o equilíbrio entre soldagem a frio e fratura foi atingido (Figura 2c). Por esse motivo, o pó com 10 horas de moagem foi utilizado na etapa de compactação e consolidação.

Para um melhor entendimento dos fenômenos que ocorreram durante a moagem de alta energia, pode-se avaliar a microestrutura da seção transversal do pó de Zamac 2 durante os diferentes tempos de moagem. Com $2 \mathrm{~h}$ de moagem, é possível identificar que as partículas encontram-se deformadas, uma vez que estas exibem uma microestrutura lamelar (Figura 3a). Ao se atingir $6 \mathrm{~h}$, observa-se que as partículas ainda apresentam lamelas e que seus formatos aproximam da forma equiaxial (Figura 3b). Depois de $10 \mathrm{~h}$, a microestrutura apresenta-se homogênea (sem lamelas) característica do estado estacionário (Figura 3c).

As densidades relativas das amostras de Zamac 2 após as etapas de compactação a frio e conformação a quente são mostradas na Figura 4 . O pó de Zamac 2 apresentou baixa compressibilidade, uma vez que após a compactação a $400 \mathrm{MPa}$, o pó atingiu 73, I\% do valor da densidade teórica. Essa baixa compressibilidade pode estar relacionada ao processo de 

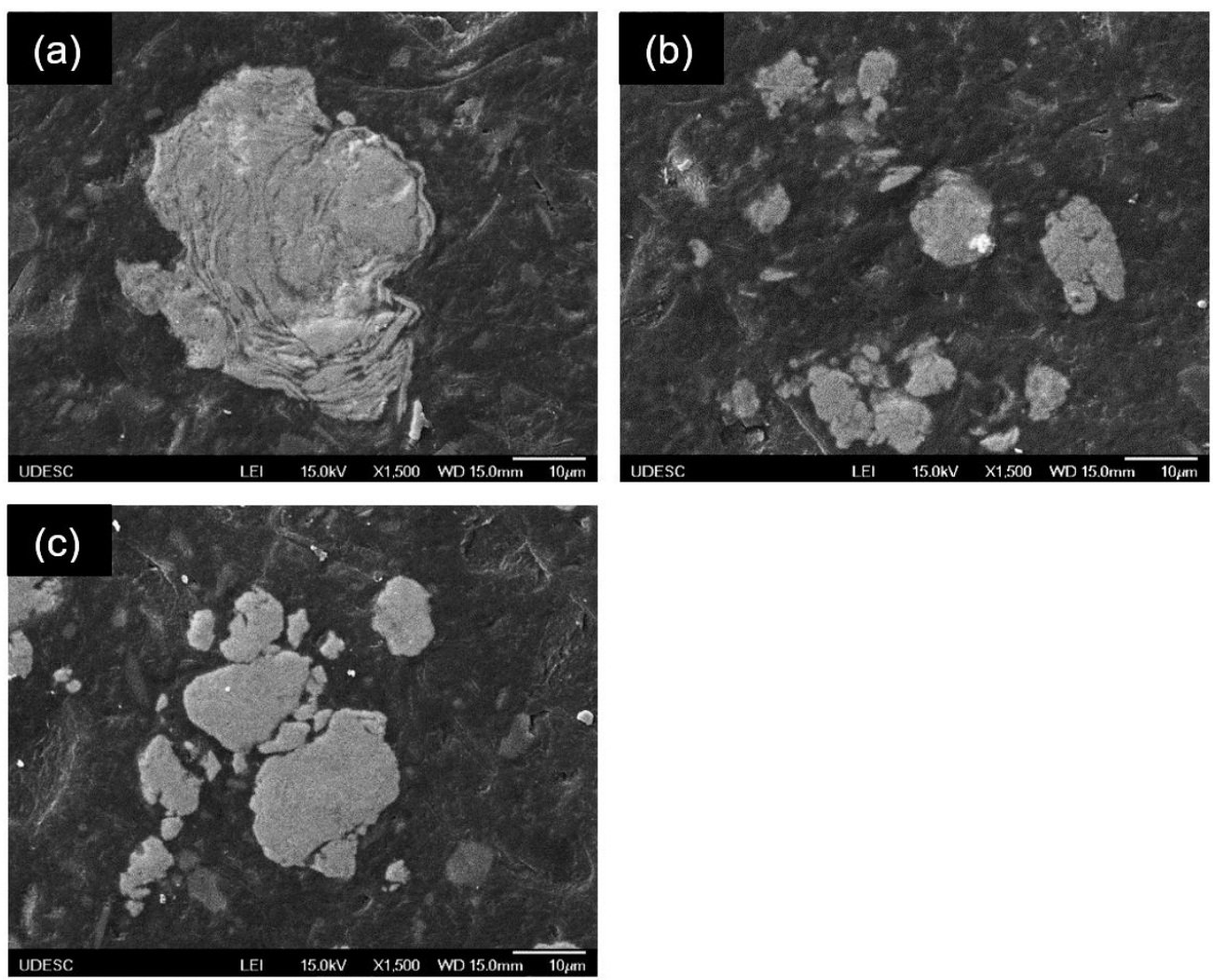

Figura 3. Microestrutura da seção transversal do pó de Zamac 2 durante a moagem: (a) 2 h, (b) 6 h, e (c) 10 h.

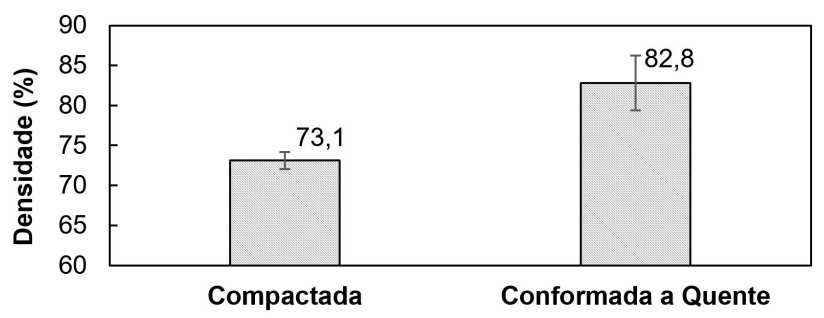

Figura 4. Densidade da Zamac 2 após a compactação e a conformação a quente.

moagem de alta energia, o qual causa aumento na resistência à deformação devido ao refino e encruamento das partículas.

$O$ processo de conformação a quente permitiu 0 aumento em torno de $13 \%$ na densidade quando comparado com a mesma condição apenas compactada (Figura 4). Esse resultado é consequência do uso simultâneo de pressão e temperatura, o qual permite o rearranjo das partículas e $\circ$ fluxo plástico nos contatos das partículas. Como resultado, há a diminuição de porosidades [9].

A microestrutura da Zamac 2 após a consolidação é mostrada na Figura 5, onde as áreas cinzas-claras são a fase $\eta$ (rica em zinco), as cinzas-escuras são a fase $\alpha$ (rica em alumínio) e os pontos brancos são os precipitados $\mathrm{CuZn}_{4}$ (fase $\varepsilon$ ). Verifica-se que a amostra apresentou coesão entre as partículas e poucas porosidades (Figura 5a). Além disso, observa-se que as fases estão distribuídas homogeneamente pelo material e que os precipitados de cobre são pequenos e com morfologia arredondada (Figura $5 b$ ).

A precipitação de $\mathrm{CuZn}_{4}$ está associada ao uso combinado de moagem de alta energia e conformação a quente. Para a produção do pó de Zamac 2 , foi adicionado $3 \%$ p de $\mathrm{Cu}$, o qual permaneceu em solução sólida devido à moagem. No entanto, ao se realizar o aquecimento do material durante a consolidação, a temperatura utilizada forneceu energia suficiente para que $\circ$ Cu precipitasse, formando a fase $\varepsilon$ [10].

O gráfico comparativo da dureza da Zamac 2 produzida por metalurgia do pó com o Zamac ZL4CZAP produzido por fundição é mostrado na Figura 6 . Conforme se observa, a dureza da liga produzida por metalurgia do pó é estatisticamente igual à da fundida. Esse resultado difere das constatações reportadas por Silva et al. [10], os quais verificaram que a dureza da liga Zamac 2 produzida por metalurgia do pó é aproximadamente $53 \%$ superior à da liga fundida. Os referidos autores utilizam os mesmos parâmetros para a consolidação a quente $\left(350^{\circ} \mathrm{C}-500 \mathrm{MPa}\right)$, porém a produção da liga foi por moagem em moinho planetário por 30 horas. $O$ emprego de menor tempo de moagem ( 10 horas) no presente trabalho pode ter contribuído para a formação de uma menor quantidade de fase T', a qual é transformada em fase $\varepsilon$ durante a conformação a quente. A fase $\varepsilon$, por ser dura, contribui positivamente para a dureza da liga e age como barreiras ao movimento das discordâncias. 

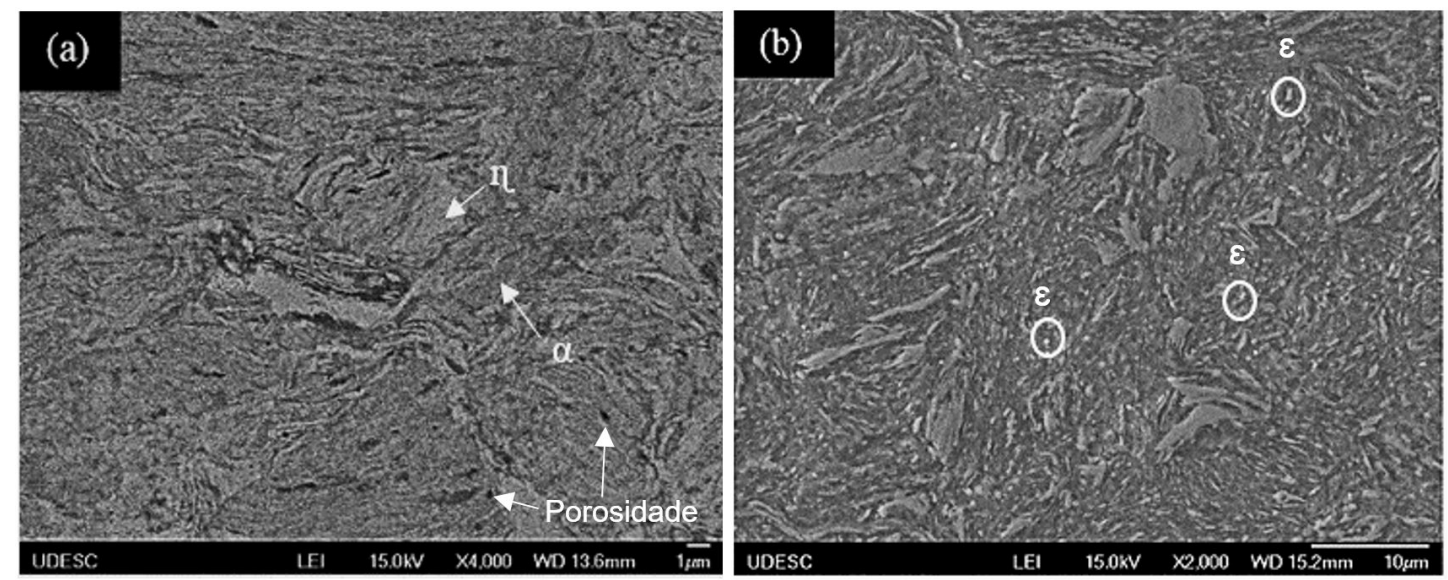

Figura 5. Microestrutura da Zamac 2 após a conformação a quente: (a) sem ataque químico e (b) com ataque Nital 3\%.

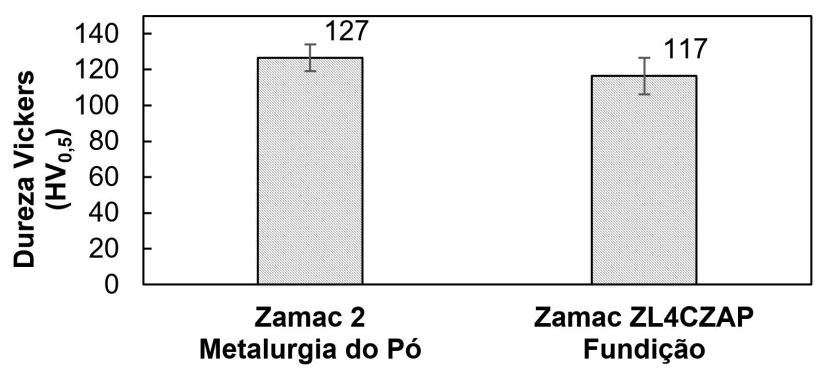

Figura 6. Dureza vickers $\left(\mathrm{HV}_{0.5}\right)$ da liga Zamac 2 produzida metalurgia do pó e da liga Zamac ZL4CZAP produzida por fundição.

\section{CONCLUSÃO}

O pó da liga Zamac 2 foi produzido via moagem de alta energia e caracterizado quanto à morfologia e à microestrutura. A densificação final, a microestrutura e a dureza de amostras de pó conformadas a quente também foram estudadas. Os resultados indicaram que dez horas de moagem em moinho atritor a $400 \mathrm{rpm}$ foram suficientes para atingir o estado de equilíbrio entre soldagem e fratura, visto que que as partículas de pó apresentaram-se homogêneas e com morfologia equiaxial. O pó de Zamac 2 apresentou baixa compressibilidade, a qual pode estar relacionada ao processo de moagem de alta energia. Verificou-se também que o uso simultâneo de pressão e temperatura durante a conformação a quente permitiu um aumento em torno de $13 \%$ na densidade, quando comparado com a mesma condição apenas compactada. Em vista disso, sugere-se o uso de taxas de deformação mais elevadas ou o uso da compactação isostática a quente (HIP) para se obter uma maior densificação nas amostras consolidadas.

Após a consolidação, observou-se que a liga apresentou coesão entre as partículas, baixa porosidade e distribuição homogênea das fases $\eta, \alpha$ e $\varepsilon$ pelo material. Além disso, os precipitados de cobre (fase $\varepsilon$ ) mostraram-se pequenos e com morfologia arredondada. Por fim, constatou-se que a dureza da liga produzida por metalurgia do pó foi estatisticamente igual à da liga fundida.

\section{Agradecimentos}

Os autores agradecem a CAPES (Coordenação de Aperfeiçoamento de Pessoal de Nível Superior) e a FAPESC (Fundação de Amparo à Pesquisa e Inovação do Estado de Santa Catarina) pelo suporte fornecido para a realização desse trabalho.

\section{REFERÊNCIAS}

I Wu Z, Sandlöbes S, Wu L, Hu W, Gottstein G, Korte-Kerzel S. Mechanical behaviour of Zn-Al-Cu-Mg alloys: deformation mechanisms of As-cast microstructures. Materials Science and Engineering A. 2016;65I:675-687.

2 Savaşkan T, Maleki RA, Tan HO. Tribological properties of Zn-25Al-3Cu-ISi alloy. Tribology International. 2015;81:105-1II.

3 Eastern Alloys Inc. Overview of zinc die casting alloys [página da internet]. [acesso em 24 abr. 20 I7]. Disponível em: Disponível em: https://www.eazall.com/overview-of-zinc-alloys

4 American Society for Testing and Materials. ASTM B86- 13: Standard Specification for Zinc and Zinc-Aluminum (ZA) alloy foundry and die castings. West Conshohocken: ASTM International; 2013. 
Proença et al.

5 Costa EM, Costa CE, Dalla FV, Rick C, Scherer M, Santos AC, et al. Study of the influence of copper and magnesium additions on the microstructure formation of $\mathrm{Zn}-\mathrm{Al}$ hypoeutectic alloys. Journal of Alloys and Compounds. 2009;488:89-99.

6 Auras R, Schvezov C. Wear behavior, microstructure, and dimensional stability of as-cast zinc-aluminum/SIC (metal matrix composites) alloys. Metallurgical and Materials Transactions. A, Physical Metallurgy and Materials Science. 2004;35: I579-1590.

7 Silva FC, Kazmierczak K, Costa CE, Milan JCG, Torralba JM. Zamak 2 alloy produced by mechanical alloying and consolidated by sintering and hot pressing. Journal of Manufacturing Science and Engineering. 20 I 7; I 39: I-7.

8 Delforge DYM, Ferreira I, Silva CGR, Paris EAG, Marcelo AL, Novaes RH. Sinterização de uma mistura de cavaco de aço inoxidável com pó do mesmo material. Uma nova tecnologia para a reciclagem de metais? Rem: Revista Escola de Minas. 2007;60:95-100.

9 Gupta N, Basu B. Hot pressing and spark plasma sintering techniques of intermetallic matrix composites. In: Mitra R, editor. Intermetallic matrix composites. USA: Elsevier; 2018. p. 243-302.

I0 Silva FC, Kazmierczak K, Costa CE, Milan JCG, Torralba JM. Zamak 2 alloy produced by mechanical alloying and consolidated by sintering and hot pressing. Journal of Manufacturing Science and Engineering. 20 I 7; I39:1-7.

Recebido em: 4 Jun. 2018

Aceito em: 10 Jul. 2019 